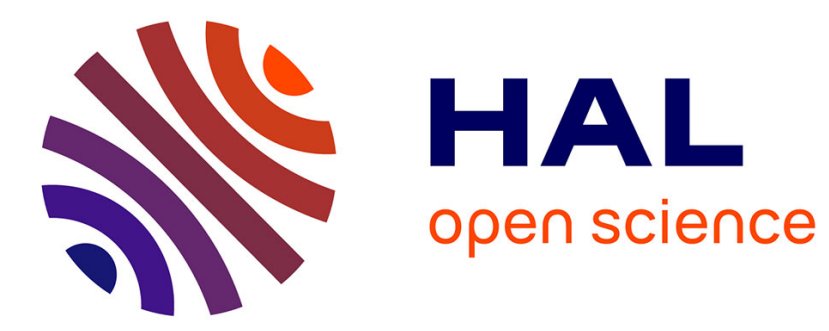

\title{
Wooki: a P2P Wiki-based Collaborative Writing Tool
} Stéphane Weiss, Pascal Urso, Pascal Molli

\section{To cite this version:}

Stéphane Weiss, Pascal Urso, Pascal Molli. Wooki: a P2P Wiki-based Collaborative Writing Tool.

[Research Report] 2007. inria-00156190v1

\section{HAL Id: inria-00156190 https://hal.inria.fr/inria-00156190v1}

Submitted on 20 Jun 2007 (v1), last revised 25 Jun 2007 (v3)

HAL is a multi-disciplinary open access archive for the deposit and dissemination of scientific research documents, whether they are published or not. The documents may come from teaching and research institutions in France or abroad, or from public or private research centers.
L'archive ouverte pluridisciplinaire HAL, est destinée au dépôt et à la diffusion de documents scientifiques de niveau recherche, publiés ou non, émanant des établissements d'enseignement et de recherche français ou étrangers, des laboratoires publics ou privés. 
INSTITUT NATIONAL DE RECHERCHE EN INFORMATIQUE ET EN AUTOMATIQUE

\title{
Wooki: a P2P Wiki-based Collaborative Writing Tool
}

\author{
Stéphane Weiss — Pascal Urso — Pascal Molli
}

$\mathbf{N}^{\circ}$ ????

Juin 2007

Thème COG

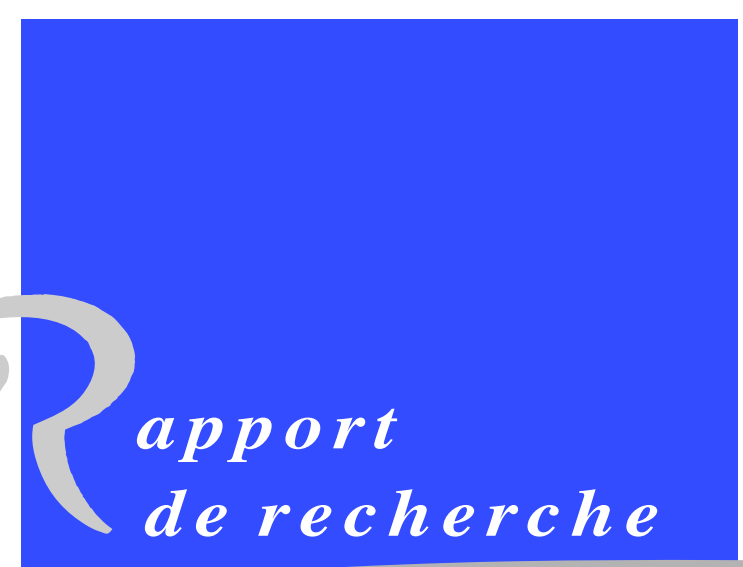





\title{
RINRIA
}

\section{Wooki: a P2P Wiki-based Collaborative Writing Tool}

\author{
Stéphane Weiss , Pascal Urso , Pascal Molli \\ Thème COG — Systèmes cognitifs \\ Projet ECOO
}

Rapport de recherche $\mathrm{n}^{\circ}$ ???? — Juin 2007 - 14 pages

\begin{abstract}
Wiki systems are becoming an important part of the information system of many organisations and communities. This introduce the issue of the data availability in case of failure, heavy load or off-line access. We propose to replicate wiki pages across a P2P network of Wiki engines. We address the problem of consistency of replicated wiki pages in the context of a P2P Wiki system. In this paper, we present the architecture and the underlying algorithms of the wooki system. Compared to traditional Wikis, Wooki is P2P Wiki which scales, delivers better performances and allows off-line access.
\end{abstract}

Key-words: P2P Wiki, Collaborative Writing, off-line access, WOOT

Unité de recherche INRIA Lorraine

LORIA, Technopôle de Nancy-Brabois, Campus scientifique, 615, rue du Jardin Botanique, BP 101, 54602 Villers-Lès-Nancy (France)

Téléphone : +33383593000-Télécopie : +33 383278319 


\section{Wooki: un éditeur collaboratif Pair à Pair basé sur un wiki}

Résumé : Les wikis deviennent une composante importante des systèmes d'information de nombreuses organisations. Ceci soulève le problème de la disponibilité des données en cas de panne, de surcharge ou d'accès hors-ligne. Nous proposons de répliquer les pages wiki sur un réseau pair à pair. Nous nous intéressons au problème de cohérence des pages wiki répliquées dans le contexte d'un système de wiki Pair à Pair. Dans ce papier, nous présentons l'architecture et les algorithmes du système wooki. Comparé aux wikis traditionnels, Wooki est un wiki Pair à Pair qui permet le passage à l'échelle, offre de meilleures performances et autorise le travail hors-ligne.

Mots-clés : Wiki P2P, Édition collaborative, accès hors-ligne, WOOT 


\section{Introduction}

Currently, Wikis are the most popular collaborative editing systems. They allow people to easily create and modify content on the web. This ease of interaction and operation makes a wiki an effective tool for collaborative writing. Collaborative writing is becoming increasingly common; often compulsory in academic and corporate work. Writing scientific articles, technical manuals and planning presentations are a few examples of common collaborative writing activities.

A lot of critical data are now under the control of wiki systems. Wiki is now used within enterprises or organizations. For example, United States intelligence community use Intellipedia for managing national security informations. Wikis are now an important piece in the information system of many organizations and communities. This introduce the issue of data availability in case of failure, heavy load or off-line access.

Current Wiki systems are intrinsically centralized. Consequently, in case of failure or off-line work, data are unavailable. In case of heavy load, system scales poorly and the cost linked to underlying hardware cannot be shared. Our objective is to replace the centralized architecture of a wiki server by a $\mathrm{P} 2 \mathrm{P}$ network of wiki servers. This make the whole wiki system fault tolerant, this allows to balance the load on the network and finally costs of the underlying hardware can be shared between different organization.

This approach suppose that Wiki data are replicated on P2P network of wiki sites. Consequently, the main problem is how to manage replicate consistency between wiki sites. Traditional pessimistic replication approaches ensure consistency but are not adapted to this context. They scale poorly and do not support off-line work.

Optimistic replication approaches suppose to know how to safely merge concurrent updates. Some previous work tried to build a $\mathrm{P} 2 \mathrm{P}$ wiki $[1,2]$ relying on distributed version control system (DVCS) approaches. The main problem with DVCS approach is correctness. An optimistic replicated system is considered as correct if it eventually converge i.e. when the system is idle all Wiki sites contain identical data. This is called eventual consistency [3]. Version control system have never ensured this property. Consequently, building a P2P system with traditional DVCS will not ensure eventual consistency. Other approaches ensure convergence $[4,5]$ but are not compatible with P2P networks constraints. Finally some others approaches converge and are adequate with $\mathrm{P} 2 \mathrm{P}$ constraints but does not support collaborative editing constraints.

We developed the woot [6] algorithm to manage consistency of replicated linear structure in a $\mathrm{P} 2 \mathrm{P}$ environment. This algorithm ensure convergence without managing versions. In this paper, we describe how we built wooki. Wooki is a fully functional P2P wiki system based on this algorithm. We refined the original algorithm to achieve a better time complexity. We combined this new algorithm with an probabilistic dissemination algorithm for managing updates propagation on the overlay network and with an anti-entropy algorithm for managing failures and disconnected sites. Wooki is currently available under GPL license.

RR $\mathrm{n}^{\circ} 0123456789$ 


\section{The Wooki approach}

A wooki network is dynamic p2p network where any site can join or leave at any time. Each site has a unique identifier named siteid. Site identifiers are totally ordered. Each site replicates wiki pages of other sites. Each site only requires a partial knowledge of the whole network.

A wooki site generates a sequence of operations when a wooki user saves its page. These operations are integrated locally on the wooki site page by executing the wooto algorithm, and broadcasted for integration on all other sites.

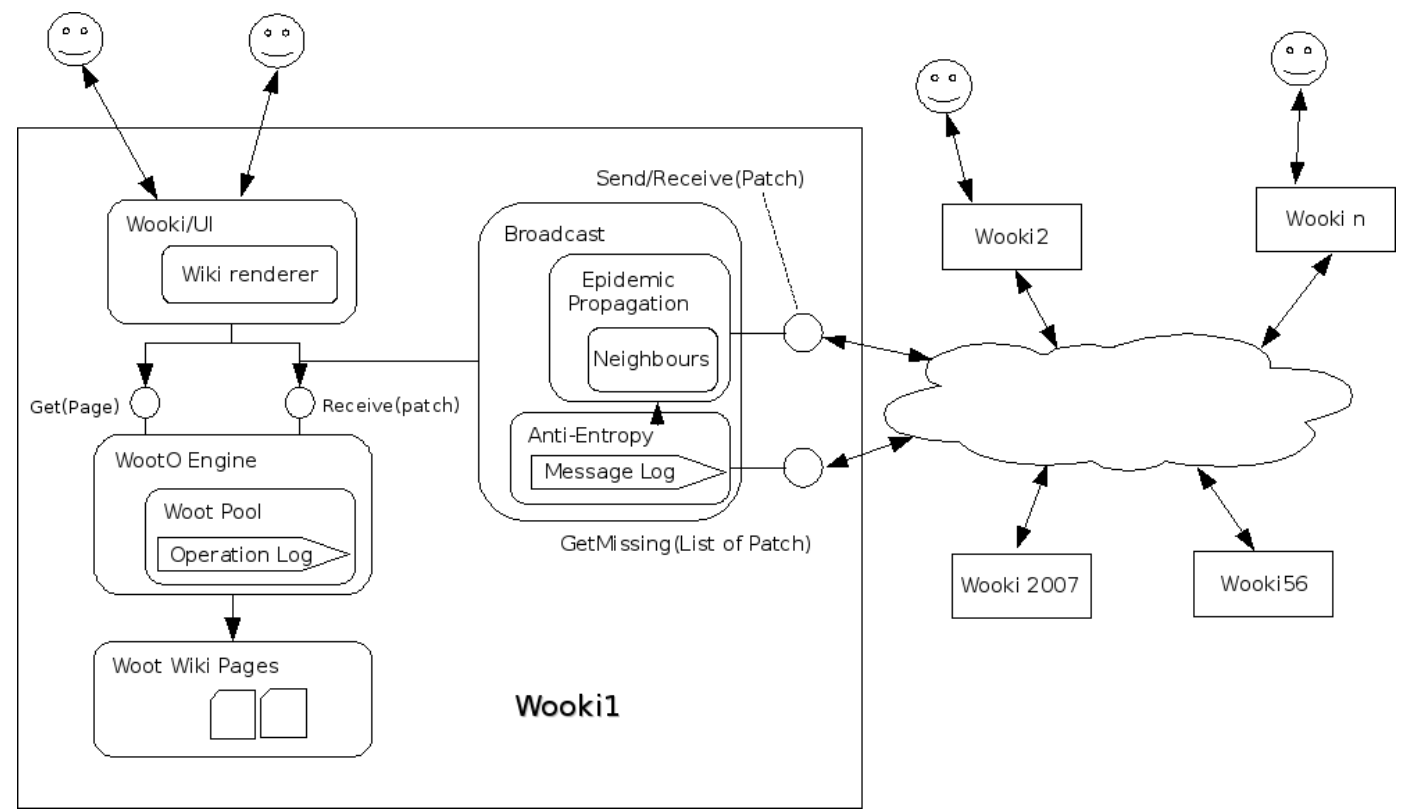

Fig. 1. Wooki architecture

Thus, there is three main components in a wooki site (figure 1). The core component wooto which is in charge of generating and integrating operations affecting the document. An other component in charge of user interface. The last component is in charge of disseminating local operations and retrieving remote operations.

\subsection{Wooto Approach}

Wooto is an optimized version of woot [6]. As woot, it ensures eventual consistency. A wooki page is identified by a unique identifier pageid. This identifier is set when the page is created. 
A wooki page contains a sequence of four-tuples $<i d l$, content, degree, visibility $>$, where each tuple represents a line of the wooki page.

$i d l$ is the unique identifier of the line. This id is a pair (siteid,logicalclock). Each site maintains a logical clock [7]. Each time an operation is generated, the logical clock is incremented. The line identifiers are strictly totally ordered.

Let idl1 and idl2 be two line identifiers with their respective values $(s 1, l 1)$ and $(s 1, l 2)$. We get $i d l 1<{ }_{i d}$ idl 2 if and only if (1) $s 1<s 2$ or (2) $s 1=s 2$ and $l 1<l 2$.

content is a string representing the content of the wiki-readable line.

degree is an integer used by the wooto algorithm. The degree of a line is fixed when the line is generated. We will describe how the degree is computed when we will describe the generation of an operation.

visibility is a boolean representing if a line is visible or not. It means that in the wooki approach, we do not delete lines, we mark them as invisible.

By applying this storage model, if we have the following wiki page:

$===$ Three pigs $==$

* [[ Image:pig.png $\mid$ thumb $\mid$ left $|100 \mathrm{px}|$ riri $]]$

fifi and loulou

Assuming these three lines were generated on site number 1 in this order and assuming there is no invisible lines, the wiki page will be internally stored as:

$((1,1),===$ Three pigs $===, 0$,true $)$

$((1,2), * \quad[[$ Image:pig. png $\mid$ thumb $\mid$ left $|100 \mathrm{px}|$ riri $]], 1$, true $)$

$((1,3)$, fifi and loulou, 2 , true $)$

To manage the storage model, the wooki handles two operations:

1. Insert(pageid, line $\left., l_{P}, l_{N}\right)$ where

- pageid is the page where to insert the line

- line $=<i d l$, line, degree, visibility $>$ is the line to be inserted.

$-l_{P}$ is the id of the previous line. This is the line after which the line must be inserted.

$-l_{N}$ is the id of the next line. This is the line before which the line must be inserted.

2. Delete (pageid, $i d l)$ set visibility of the line identified by $i d l$ as false in the page identified by pageid. Optionally, the content of the deleted line can be garbaged. However, to maintain consistency, the deleted line identifier must be kept as tombstones. Tombstones, also known as "death certificate", are heavily used in optimistic replication, especially in Usenet [8] or replicated databases [9].

The main issue with tombstones is how to garbage them. Several propositions have been made in [9], but they are not adapted to the P2P context. In wooto, we decided to let the model grows infinitely. We motivate this choice by the need in collaborative editing to keep trace of all changes. Wikipedia, for instance, makes the same choice and keeps all versions of every articles.

RR nº 0123456789 
When woot creates an new page, the page is initialized as : $L_{B}, L_{E}$. Where $L_{B}$ and $L_{E}$ are special lines indicating the beginning and the ending of a page. When site $x$ generates an insert operation on page $p$, between line $A$ and line $B$, it generates the operation

$$
\operatorname{Insert}\left(p,<\left(x,++\operatorname{clock} k_{x}\right), \text { content }, d, \text { true }>, \operatorname{idl}(\text { line } A), i d l(\text { line } B)\right)
$$

Where $d=\max (\operatorname{degree}($ line $A)$, degree $($ line $B))+1$. By definition, $L_{B}$ and $L_{E}$ have a degree of 0 .

Every generated insert operations must be integrated on every wooki sites (including the generation one). The wooto algorithm is able to integrate insert operations and to compute the same result whatever the integration order of insert operations. In the following algorithm, $S$ represents, on the local site, the lines sequence of the page where a line have to be inserted.

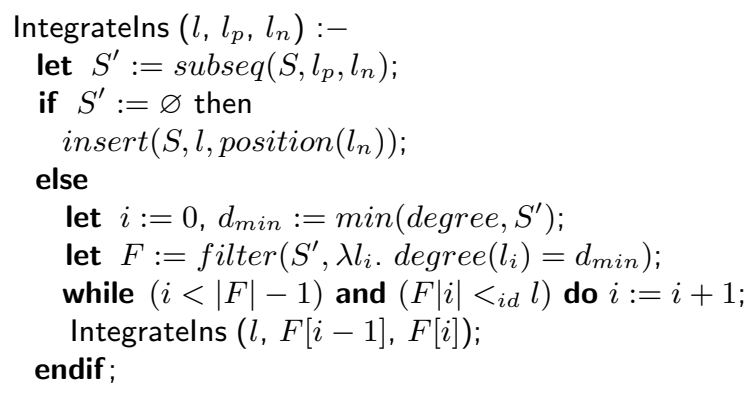

This algorithm selects the sub-sequence of lines present between the previous line and the next line. If this sequence is empty, the line $l$ is inserted in the model just before the next line. Elsewhere, wooto filters the sub-sequence keeping only lines with the minimum degree. The remaining lines are sorted according the $<_{i d}$ order [6]. Thus, $l$ must be integrated at its place according $<_{i d}$ between remaining lines. We then make a recursive call to place $l$ among lines with higher degree.

However, since wooki sites can receive operations in any order, the operations have preconditions. When a site receives an operation, if its pre-condition are verified, the operation can be integrated immediately by executing the wooto algorithm. If pre-condition are false, the operation is placed on a waiting queue until its pre-condition are verified. Pre-conditions are: a line can only be inserted on a site if its previous and next lines are already present in the model of this site. Similarly, only an existing line can be deleted.

Let's now illustrate wooto algorithm through an example (see figure 2). On this scenario, line 1 and line 2 were concurrently inserted when the page was empty, and line 3 was inserted before line 1. Without woot or wooto, such a scenario can lead to three different line orders $L_{B}, 2,3,1, L_{E}, L_{B}, 3,2,1, L_{E}$ or $L_{B}, 3,1,2, L_{E}$ each of them respecting the previous and next relationship. We show how wooto computes independently the same order $L_{B}, 3,1,2, L_{E}$ on every wooki site. 


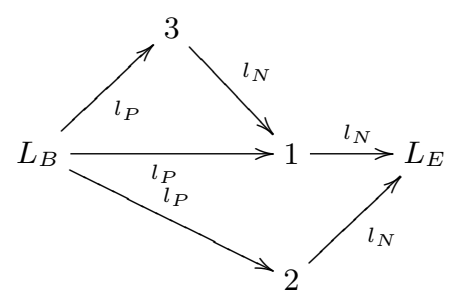

Fig. 2. Example

According to definition, we have degree $(1)=\operatorname{degree}(2)=1$ and degree $(3)=2$. Due to pre-conditions, there is no site where line 3 is present and not line 1 . Now, let's assume that $1<_{i d} 2<_{i d} 3$.

- Imagine a site A where line 2 arrives when only line 1 is present (and not yet line 3 ). The wooto algorithm first selects the lines present between $L_{B}$ and $L_{E}$. Since there is only the line 1 , line 2 is placed after to obtain $L_{B}, 1,2, L_{E}$. When line 3 arrives, it is placed between $L_{B}$ and line 1 to obtain $L_{B}, 3,1,2, L_{E}$.

- Similarly, if line 1 arrives when line 2 is present, it is placed before to obtain $L_{B}, 1,2, L_{E}$ and finally $L_{B}, 3,1,2, L_{E}$.

- Now, imagine a site B, where line 2 arrives when line 1 and line 3 are present. The wooto algorithm first selects the lines present between $L_{B}$ and $L_{E}$ and filters them to keep only line 1 . The reason of such a filtering is above. Indeed, since line 3 is dependent to an other line - here line 1 -, it has an higher degree. Moreover, there could exist a site (such as site A) where line 1 is present and not line 3 . Thus, line 2 must be placed according to line 1 earlier to line 3 . Site B also obtains $L_{B}, 3,1,2, L_{E}$.

Finally, compared to woot original integration algorithm, the filtering is done on degree instead of the previous and next relationship of each line. Thus, wooto has a better time complexity : $O\left(n^{2}\right)$ instead of $O\left(n^{3}\right)$. This also allows to reduce the space required to store the lines: an integer replacing two line identifiers. As woot, we have formally verified the eventual consistency of wooto with the Lamport's model-checker TLC [10].

\section{$2.2 \quad$ User's operation}

The users do not directly edit the model. When an user opens a page for editing, he sees a view of the model which presents only the content of visible lines. As in traditional wiki, the user makes all the modifications he wants and saves.

To detect operations, we use a diff algorithm [11] between the page the user requested at edition time and the page the user saves. We translate the operations given by the diff algorithm in terms of wooto operations. A delete of the line number $n$ is translated into a delete of the $n^{t h}$ visible line. An insert at position $n$ is translated into a insert between the $(n-1)^{t h}$ and the $n^{t h}$ visible lines. These operations are integrated locally and broadcasted to the other wooki sites.

$\mathrm{RR} \mathrm{n}^{\circ} 0123456789$ 
Weiss, Urso and Molli

\subsection{Wooki Broadcast}

For operation dissemination, we use a broadcast protocol in the spirit of [12]. We combine a probabilistic broadcast algorithm with an anti-entropy algorithm [9]. The probabilistic broadcast quickly disseminates updates on the P2P network and to manage membership. The anti-entropy algorithm has the responsibility to recover missing updates for sites that were off-line or crashed.

In order to be deployed on a $\mathrm{P} 2 \mathrm{P}$ network, the broadcast protocol must be scalable, reliable and must also support the churn of the network. Indeed, in a P2P network, nodes join and leave the network dynamically. Thus, we replace the standard probabilistic broadcast of [12] by the lightweight probabilistic broadcast (lpbcast) [13]. This algorithm gives probabilist warranties that sent operations will be delivered to all connected nodes. When a site receives a message:

1. it delivers the message to wooto,

2. it decreases the number of rounds attached to this message,

3. it forwards the message to its neighbors. If a site receives a message with a null round, it stops propagation of the message.

In order to ensure a reliable dissemination of messages, each site must manage a table of neighbors. This table has a fixed size and contains only a partial nodes list of the entire P2P network. Lpbcast updates this table during messages propagation. Some subscriptions and unsubscriptions attached to each message are used to update table of neighbors. Lpbcast gives probabilistic warranties that there is no clusters within P2P network. Moreover, since wooto does not require an ordering on message reception, the lpbcast can be unordered for higher efficiency.

The lpbcast algorithm ensures a reliable and scalable dissemination of operations on connected nodes. For managing off-line work, we combine it with an anti-entropy algorithm. We use the original anti-entropy algorithm of [9]. When a site starts an anti-entropy, it selects a neighbor at random in the local table of neighbors and send to him a digest of his own received messages. The selected site returns missing messages to caller. Using antientropy implies that each site keeps received messages in a log. As this log cannot grow infinitely, we purge this log from time to time. Purging the log in an intrinsic problem of anti-entropy approach. If a site purges its $\log$ and then starts an anti-entropy with another site, it can receive previously purged messages. In the traditional approach, tombstones or death certificates are used to avoid this problem.

Fortunately, Wooki do not need to use such message tombstone since it supports reintegration of already integrated operations. Indeed, we drop an insert operation of a line which identifier is already present in the model. Also, reapplying deletion of a line causes no modification. Thus, we can purge the anti-entropy log without using operation tombstones. This is an interesting property of combining wooto and anti-entropy approach.

Finally, if a site is off-line for a long period of time, anti-entropy may not find missing messages i.e. missing messages which have been purged on all sites of the P2P network. In this case, this site cannot be synchronized. The only way to recover this site is to make a 
state transfer with a site. It is important to notice that, even in this case, off-line work is not lost since local operations are still applicable. The site can send its local operations and then make the state transfer.

\section{Implementation}

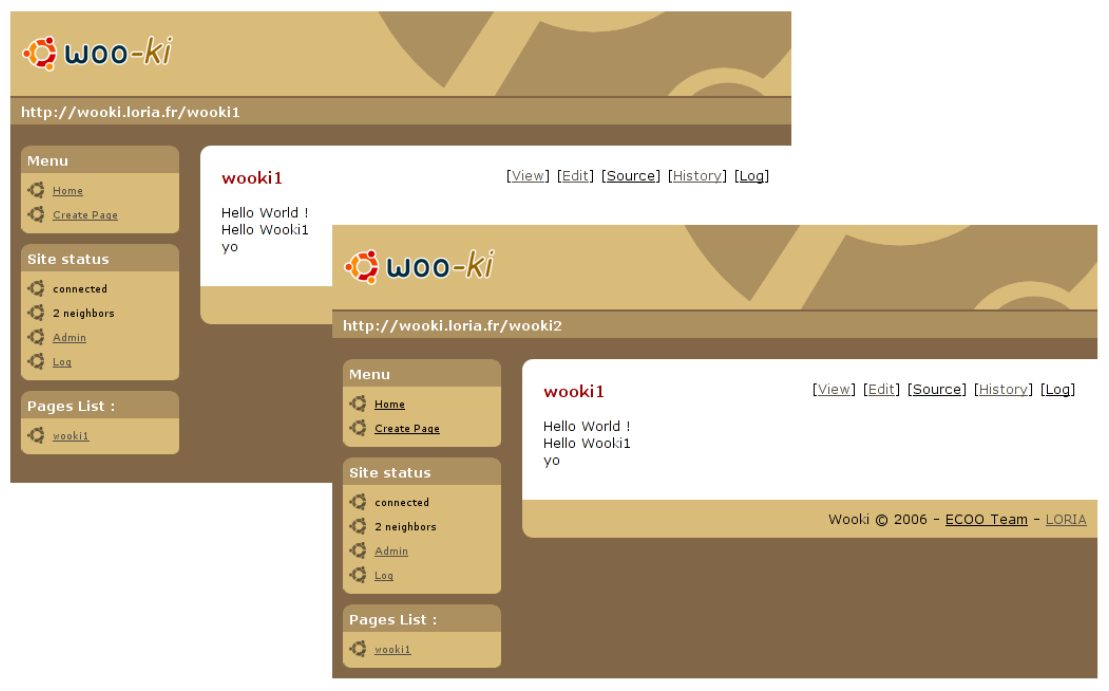

Fig. 3. Wooki Interface

The Wooki prototype has been implemented in Java as servlets in a Tomcat Server. Wooki pages are just stored in regular files. All network messages are transported by the http protocol. In figure 3, we can see the same page entitled "wooki1" loaded from 2 different Wooki sites:

- http://wooki.loria.fr/wooki1

- http://wooki.loria.fr/wooki2

In this example, the same server "wooki.loria.fr" hosts 3 wooki sites. We can also see on figure 3 that both sites are connected and each site has two neighbors. We can observe the table of neighbors in figure 4 . This interface let the administrator manage manually the routing table. In the normal case, no administration is required except when starting wooki for the first time. The administrator has to connect the new wooki site to an existing wooki network by entering the http address of an wooki node. Once bootstrapped, the routing table is updated when messages from other sites are received.

$\mathrm{RR} \mathrm{n}^{\circ} 0123456789$ 


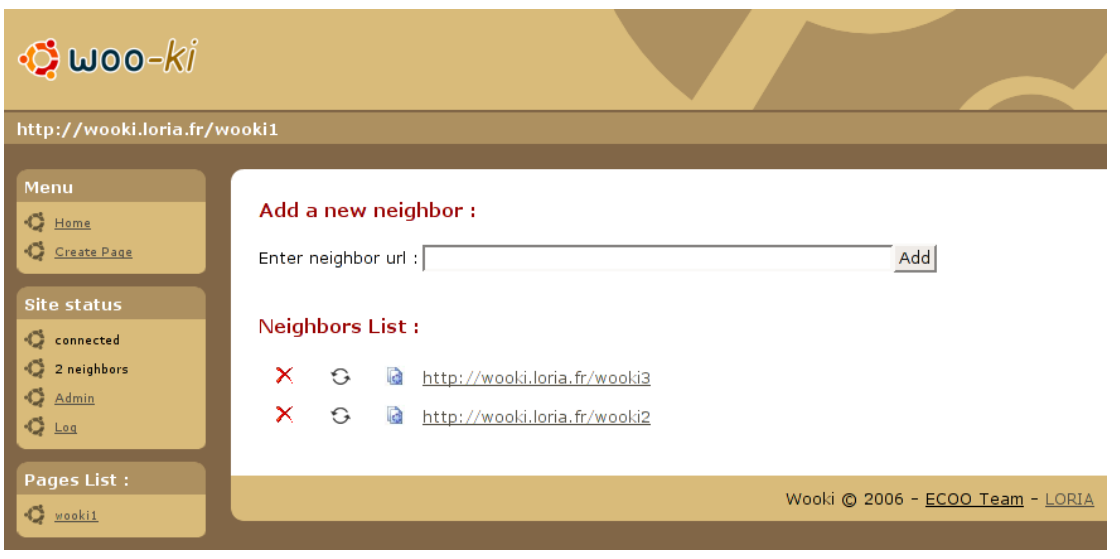

Fig. 4. Wooki Neighbors

This interface also allows the administrator to start anti-entropy or state transfer. In the normal case, anti-entropy is activated at regular interval of time. Administrator can force anti-entropy when he restarts the wooki system or when an off-line session is ended.

The wooki prototype is available with a GPL license at http://p2pwiki.loria.fr.

\section{Related work}

The pessimistic replication (database approach [14] or consensus [15]) ensures that, at anytime, all replicas host the same content. Pessimistic replication is widely used and recognized for its safety. Unfortunately, pessimistic replication is also well-known for its poor scalability [3] and its incapacity to provide an off-line work.

Virtual Synchrony [16] is a middleware which allows sharing data among programs running on multiple machines. Virtual Synchrony provides a pessimistic replication with some optimizations to improve global performance. Consistency is ensured by enforcing a total ordered reception of modifications. Unfortunately, this ordering is costly and do not scale to a huge number of sites. In addition, Virtual Synchrony does not allow disconnected work.

Icecube [17] is an optimistic reconciliation algorithm. Using static or dynamic reconciliation constraints, some semantic relations can be defined between users' operations. The Icecube algorithm aimed to obtain the best operations' schedule satisfying all constraints. Therefore, Icecube requires a central site which have to choose this schedule. Unfortunately, a central site is a serious bottleneck, hence, Icecube is not well-fit to provide a scalable wiki. In addition, due to concurrency, some constraints may not be satisfiable, hence, some operations have to be dropped. On the contrary, in the woot approach, operations never conflict and, consequently, they are all integrated.

Joyce [5] is a programing framework based on the Icecube approach. In Joyce, the schedule is incrementally determined. Using Joyce, the authors proposed a collaborative text editor 
called Babbles which supports selective undo/redo mechanism. Thanks to the constraints, babbles can detect insertion conflicts. On the contrary, we consider that two insertions never conflict. The main drawback of Babbles is the requirement of a primary site which have to resolve conflicts. This primary limits the scalability, and is a single point of failure.

The operational transformation (OT) approach [4] is composed by operations which express modifications, and the transformation functions used to modify concurrent operations toward local operations. A state vector is associated to each operations. The state vector size grows linearly with the number of sites. Consequently, the state vector's size limits the scalability of this approach. The woot approach depends only of the number of operations and not of the number of sites.

Distributed version control systems (DVCS) allow many users to edit the same documents concurrently. They provide the same features as CVS [18] or Subversion [19] without requiring a central site. DVCS do not express the notion of consistency. In addition, a wellknown scenario (Figure 5) from the OT approach [20] leads most of DVCS (Darcs, Mercurial, Git, Bazaar, ...) to the document inconsistency. In order to reproduce a such scenario with DVCS, we use three branches to play the sites' role. Hence the document's state is respectively "aXbc", "ac", "abYc" according to this three branches. A workspace is associated to each branches. In the OT approach, a site receives other sites' operations. Here, a workspace user makes an update from an other workspace. Code Co-op [1] is a DVCS which allows to add a wiki to a replicated folder. Hence, we obtain a p2p wiki. As many DVCS, Code Co-op cannot ensures the wiki's pages consistency.

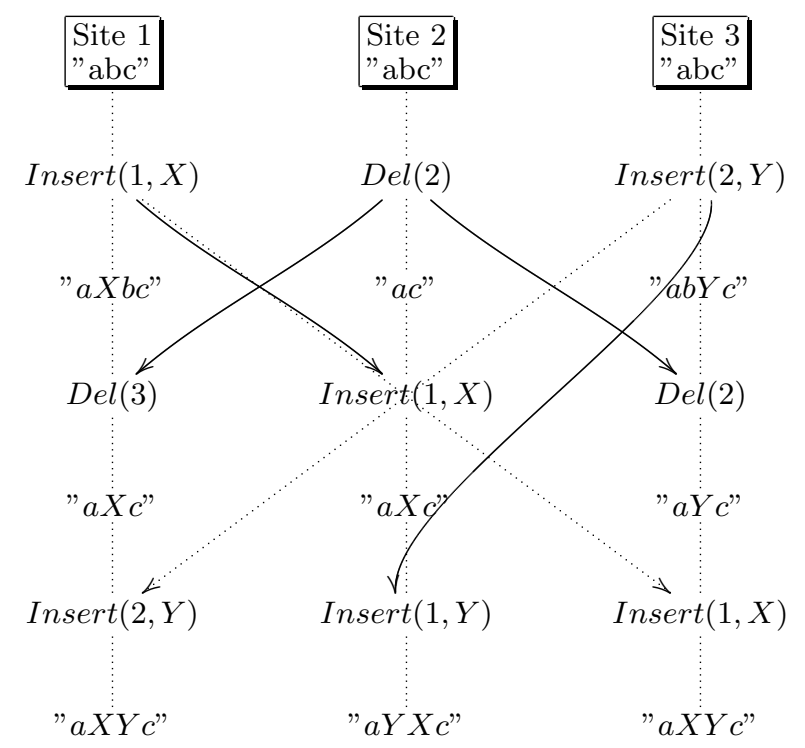

Fig. 5. Divergence scenario from the OT approach.

RR nº 0123456789 
RepliWiki [2] is a P2P wiki based on the SHH approach [21]. Each site hosts a replica of all wiki pages. To each wiki pages, an history graph is associated. In this history, a version is represented by a unique identifier obtained by using a hash function. Like wooki, RepliWiki uses an anti-entropy mechanism to propagate modifications.

Repliwiki requires a correct merge algorithm to reconciliate concurrent modifications. Consequently, it uses the SHH-sync which is a DVCS. Unfortunately, as claimed before, DVCS algorithms failed to ensure consistency. In other words, whenever the SHH approach correctly detects causality and concurrency, repliwiki cannot ensure consistency using existing merge algorithms. In addition, a site's log grows indefinitely. A prune procedure is proposed, it allows a site to brutally truncate his hash history but requires at least one archiver site which never purge its log. In such a configuration, the reliability is weakened. If all archiver sites crashed, the consistency could not be ensured.

\section{Conclusion}

Wooki is a P2P wiki system. Compared to traditional centralized wikis, a P2P wiki system is fault-tolerant, improves global performances and allows user to work off-line. Wooki uses the wooto algorithm to manage consistency of copies. Wooto is an optimized version of the woot algorithm and ensures eventual consistency. Compared to other optimistic replication approaches, wooto is designed for $\mathrm{P} 2 \mathrm{P}$ networks and is fully compatible with $\mathrm{P} 2 \mathrm{P}$ network constraints.

In this paper, we described how to combine the wooto algorithm with an epidemic dissemination algorithm. This combination provides a reliable $\mathrm{P} 2 \mathrm{P}$ wiki system that scales and tolerates the "churn" of P2P networks. It also supports long-term disconnections without ever loosing off-line work.

The Wooki system has several open issues:

- We clearly make the choice to keep all tombstones in the wooto page model. It implies that wooki pages will grow infinitely. Nevertheless, keeping a tombstone has a very small space overhead. In the context of a wiki system, this choice is reasonable. In another context, it is interesting to design a distributed tombstone garbage collector that is compatible with $\mathrm{P} 2 \mathrm{P}$ constraints.

- A P2P wiki system changes interactions between humans and the system. In a central wiki system, a user is aware about concurrent changes when he saves its page. If the wiki page has changed during the edition, the user have to resolve conflicts before committing his changes. In a P2P wiki system, a user can save its changes before concurrent ones arrive for integration. Wooto will solve the conflicts but the final page available on the site is a page computed by the system but not reviewed by a human. We need to add to wooki an awareness engine that is responsible to warn about concurrent changes.

- Replicating a Wiki site is not completely transparent. If a wiki page contains some macros that computes some statistics, the visible result will not be the same on all sites. Suppose a counter that count the read number of a page. If the page is replicated, 
this counter can have different values on different sites. This does not violate eventual consistency. The source page is still the same on all sites, but the rendering of the page can be different.

- In collaborative editing, it is important to undo some operations. In traditional Wikis, it is possible to revert to a previous version of the wiki. In the general case, it must be possible to undo any operations (not always the last one), anytime [22]. We have not yet provided such features in wooto. However, as we keep all informations within pages, it should be possible to undo an operation. We still need to design such an algorithm and prove eventual consistency in case of undo.

- In Wooki, we built a new P2P wiki system. We are working on integration of the Wooki engine with existing Wiki systems. The general approach is to manage optimistic replication by only using the web service interface of existing Wiki systems.

\section{References}

1. Reliable Software: Code Co-op. (2006) http://www.relisoft.com/co_op.

2. Kang, B.B., Black, C.R., Aangi-Reddy, S., Masri, A.E.: Repliwiki: A next generation architecture for wikipedia. (Unpublished) http://isr.uncc.edu/repliwiki/repliwiki - conference.pdf

3. Saito, Y., Shapiro, M.: Optimistic replication. ACM Computing Surveys 37(1) (2005) 42-81

4. Ellis, C.A., Gibbs, S.J.: Concurrency control in groupware systems. In: SIGMOD Conference. Volume 18. (1989) 399-407

5. O'Brien, J., Shapiro, M.: An application framework for nomadic, collaborative applications. In Eliassen, F., Montresor, A., eds.: DAIS. Volume 4025 of Lecture Notes in Computer Science., Springer (2006) 48-63

6. Oster, G., Urso, P., Molli, P., Imine, A.: Data consistency for p2p collaborative editing. In: Proceedings of the 2006 ACM Conference on Computer Supported Cooperative Work, CSCW 2006, Banff, Alberta, Canada, November 4-8, 2006, ACM (2006)

7. Lamport, L.: Time, clocks, and the ordering of events in a distributed system. Commun. ACM 21(7) (1978) 558-565

8. Spencer, H., Lawrence, D.: Managing Usenet. O'Reilly (January 1998)

9. Demers, A., Greene, D., Hauser, C., Irish, W., Larson, J., Shenkcr, S., Sturgis, H., Swinehart, D., Terry, D.: Epidemic algorithms for replicated database maintenance. In: Proceedings of the Sixth annual ACM Symposium on Principles of Distributed Computing - PODC'87, ACM Press (1987) 1-12

10. Yu, Y., Manolios, P., Lamport, L.: Model checking TLA+ specifications. In: Proceedings of Correct Hardware Design and Verification Methods - CHARME'99. (1999) 54-66

11. Myers, E.W.: An o(nd) difference algorithm and its variations. Algorithmica 1(2) (1986) 251266

12. Birman, K.P., Hayden, M., Ozkasap, O., Xiao, Z., Budiu, M., Minsky, Y.: Bimodal multicast. ACM Trans. Comput. Syst. 17(2) (1999) 41-88

13. Eugster, P.T., Guerraoui, R., Handurukande, S.B., Kouznetsov, P., Kermarrec, A.M.: Lightweight probabilistic broadcast. ACM Trans. Comput. Syst. 21(4) (2003) 341-374

14. Bernstein, P.A., Hadzilacos, V., Goodman, N.: Concurrency Control and Recovery in Database Systems. Addison-Wesley (1987)

$\mathrm{RR} \mathrm{n}^{\circ} 0123456789$ 
15. Lynch, N.A.: Distributed Algorithms. Morgan Kaufmann Publishers Inc., San Francisco, CA, USA (1996)

16. Birman, K.P., Joseph, T.A.: Exploiting virtual synchrony in distributed systems. In: SOSP. (1987) 123-138

17. Kermarrec, A.M., Rowstron, A.I.T., Shapiro, M., Druschel, P.: The IceCube approach to the reconciliation of divergent replicas. In: Proceedings of the twentieth annual ACM symposium on Principles of distributed computing - PODC'01, ACM Press (2001) 210-218

18. Berliner, B.: CVS II: Parallelizing software development. In: Proceedings of the USENIX Winter 1990 Technical Conference, Berkeley, California, United States, USENIX Association (1990) 341-352

19. : Subversion http://subversion.tigris.org/. June 2005.

20. Oster, G., Urso, P., Molli, P., Imine, A.: Tombstone transformation functions for ensuring consistency in collaborative editing systems. In: The Second International Conference on Collaborative Computing: Networking, Applications and Worksharing (CollaborateCom 2006), Atlanta, Georgia, USA, IEEE Press (November 2006)

21. Kang, B., Wilensky, R., Kubiatowicz, J.: The hash history approach for reconciling mutual inconsistency. In: ICDCS, IEEE Computer Society (2003) 670-677

22. Sun, C.: Undo as concurrent inverse in group editors. ACM Transactions on Computer-Human Interaction (TOCHI) 9(4) (December 2002) 309-361 


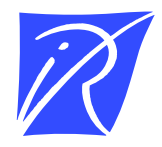

Unité de recherche INRIA Lorraine

LORIA, Technopôle de Nancy-Brabois - Campus scientifique 615, rue du Jardin Botanique - BP 101 - 54602 Villers-lès-Nancy Cedex (France)

Unité de recherche INRIA Futurs : Parc Club Orsay Université - ZAC des Vignes

4, rue Jacques Monod - 91893 ORSAY Cedex (France)

Unité de recherche INRIA Rennes : IRISA, Campus universitaire de Beaulieu - 35042 Rennes Cedex (France)

Unité de recherche INRIA Rhône-Alpes : 655, avenue de l’Europe - 38334 Montbonnot Saint-Ismier (France) Unité de recherche INRIA Rocquencourt : Domaine de Voluceau - Rocquencourt - BP 105 - 78153 Le Chesnay Cedex (France)

Unité de recherche INRIA Sophia Antipolis : 2004, route des Lucioles - BP 93 - 06902 Sophia Antipolis Cedex (France) 